\title{
Erratum: Motion of Spin-Half Particles in the Axially Symmetric Field of Naked Singularities of the Static $q$-Metric [Gravitation and Cosmology 23 (2), 149-161 (2017)]
}

\author{
Vasily P. Neznamov ${ }^{* 1,2}$ and Valery E. Shemarulin ${ }^{* * 1}$ \\ ${ }^{1}$ Russian Federal Nuclear Center-All-Russian Research Institute of Experimental Physics, \\ pr. Mira 37, Sarov, Nizhny Novgorod region, 607188 Russia \\ ${ }^{2}$ National Research Nuclear University MEPhI, Moscow, Russia \\ Received June 22, 2017
}

DOI: $10.1134 / \mathrm{S} 0202289317040156$

The following corrections are in order to the published paper [1]:

1. On page 149, Eq. (1), printed as

$$
\begin{aligned}
d s^{2} & =\left(1-\frac{r_{0}}{r}\right)^{1+q} c^{2} d t^{2}-\left(1-\frac{r_{0}}{r}\right)^{-q} \\
& \times\left[\left(1+\frac{r_{0} \sin ^{2} \theta}{4 r^{2}\left(1-\frac{r_{0}}{r}\right)}\right)^{-q(2+q)}\right. \\
\times & \left.\left(\frac{d r^{2}}{1-\frac{r_{0}}{r}}+r^{2} d \theta^{2}\right)+r^{2} \sin ^{2} \theta d \varphi^{2}\right],
\end{aligned}
$$

should read

$$
\begin{aligned}
d s^{2} & =\left(1-\frac{r_{0}}{r}\right)^{1+q} c^{2} d t^{2}-\left(1-\frac{r_{0}}{r}\right)^{-q} \\
& \times\left[\left(1+\frac{r_{0}^{2} \sin ^{2} \theta}{4 r^{2}\left(1-\frac{r_{0}}{r}\right)}\right)^{-q(2+q)}\right.
\end{aligned}
$$

$$
\left.\times\left(\frac{d r^{2}}{1-\frac{r_{0}}{r}}+r^{2} d \theta^{2}\right)+r^{2} \sin ^{2} \theta d \varphi^{2}\right] .
$$

2. On page 150, Eq. (4), printed as

$$
a(r, \theta)=\left(1+\frac{r_{0} \sin ^{2} \theta}{4 r^{2} f_{S}}\right)^{-q(2+q)},
$$

should read

$$
a(r, \theta)=\left(1+\frac{r_{0}^{2} \sin ^{2} \theta}{4 r^{2} f_{S}}\right)^{-q(2+q)} .
$$

The above corrections do not affect the results.

\section{REFERENCES}

1. Grav. Cosmol. 23 (2), 149-161 (2017), doi $10.1134 / \mathrm{S} 0202289317020050$

*E-mail: neznamov@vniief.ru

**E-mail: shemarulin@vniief.ru 